\title{
Mapas conceptuales: reflexión educativa desde la concepción constructivista sociocultural del aprendizaje
}

\section{Flor Jiménez Segura}

Docente e investigadora del Instituto de Investigación en Educación de la Universidad de Costa Rica. Orientadora de la Universidad Estatal a Distancia de Costa Rica. Licenciada en Ciencias de la Educación con énfasis en Orientación, MSc. en Gerontología por la Universidad de Costa Rica, Magister en Psicología Educativa por la Universidad de Barcelona y Doctoranda de Psicología Educativa por la Universidad de Barcelona; fimenez@uned.ac.cr.

Recibido: 26 de abril de 2010

\section{RESUMEN}

La concepción constructivista sociocultural del aprendizaje no ha dejado de lado el papel que dentro del proceso de enseñanza y aprendizaje pueden aportar las tecnologías de la información y la comunicación (TIC), ya que desde este enfoque no son vistas como algo aislado al proceso de aprendizaje ni como medios que transmiten información y propician la sustitución del educador. Todo lo contrario, se promueven como herramientas semióticas o instrumentos psicológicos que pueden influir en los procesos de construcción de significados y de atribución de sentido que llevan a cabo las personas durante la enseñanza y el aprendizaje. A partir de la perspectiva constructivista de orientación sociocultural se pretende describir la contribución que el software CmapTools puede brindar al proceso de enseñanza y aprendizaje, concretamente al ajuste de la ayuda del docente, así como su participación en el proceso de autorregulación individual y grupal en situaciones de aprendizaje colaborativo.

\section{PALABRAS CLAVE}

Perspectiva constructivista, sociocultural, CmapTools, ajuste de la ayuda, autorregulación.
Aceptado: 16 de agosto de 2010 psychological tools can enhance the development of meanings that occur during teaching and learning. The contribution of the CmapTools software is described, from a constructivist-sociocultural perspective. It is seen as a psychological instrument that aids the learning process, specifically the teachers' support adjustment and his/her participation in group as well as self-regulation in collaborative learning situations.

\section{KEY WORDS}

Constructivist sociocultural, perspective, CmapTools, support adjustment, self-regulation. 


\section{INTRODUCCIÓN}

La perspectiva constructivista sociocultural y situada del proceso de enseñanza y aprendizaje entiende la educación como una práctica social con fines de socialización e individualización, como un instrumento por el cual una sociedad intenta promover el desarrollo de las personas por medio del aprendizaje de saberes culturales relevantes que respondan a sus intenciones educativas, las cuales se concretan en un currículo que incluye contenidos y conocimientos fundamentales para el desarrollo de las competencias necesarias de convivencia social; "las intenciones educativas se logran a partir de una ayuda sistemática, planificada y continuada por parte de los adultos o personas más preparadas" (Coll, 2001: 157).

La finalidad de la educación es promover la construcción de la identidad personal y la socialización de las generaciones más jóvenes, que las sitúe de forma competente, participativa, activa y provechosa en la sociedad, a fin de que ésta logre sus expectativas sobre la educación, la adquisición de aprendizajes básicos imprescindibles y la inclusión social; de esta forma, muy presumiblemente estaríamos ante un sistema educativo de calidad.

Desde la perspectiva constructivista sociocultural, el aprendizaje escolar se da a partir de la interacción entre los alumnos y el profesor -quien ayuda a los alumnos a construir significados y a atribuir sentido a lo que hacen y aprenden, construyendo su propio conocimiento y aprendiendo a aprender-. Se trata de una actividad cuyos componentes fundamentales deben contemplarse y explicarse gracias a las relaciones o influencias mutuas que, en el ámbito del aula, se explican en términos de construcción, mediación e internalización (Vygotsky, 1979).

"Lo que los alumnos aportan al acto de aprender, su actividad mental constructiva, es un elemento mediador entre la enseñanza del profesor y los aprendizajes que llevan a cabo. Recíprocamente, la influencia educativa que ejerce el profesor a través de la enseñanza es un elemento mediador entre la actividad mental constructiva de los alumnos y los significados que vehiculan los contenidos escolares." (Coll, 2001: 179).

Por tanto, la enseñanza es un proceso de ajuste para la ayuda educativa que hace el docente, el cual supone la diversificación de los distintos apoyos y la flexibilidad en los diferentes momentos del proceso educativo, para lograr la atención a la diversidad y el aprendizaje de los alumnos, de un proceso de construcción de la actividad mental cognitiva generadora de significado y sentido.

La concepción constructivista sociocultural del aprendizaje no ha dejado de lado el papel que dentro del proceso de enseñanza y aprendizaje pueden aportar las tecnologías de la información y la comunicación (TIC), que desde este enfoque no son vistas como algo aislado al proceso de aprendizaje ni como medios que transmiten información y propician la sustitución del educador. Todo lo contrario, se promueve el uso de las TIC como herramientas semióticas o instrumentos psicológicos, las cuales pueden influir en los procesos de construcción de significados y de atribución de sentido que llevan a cabo las personas durante la enseñanza y el aprendizaje, "así como los mecanismos de influencia educativa que facilitan, promueven y apoyan estos procesos constructivos" (Coll y Martí, 2001: 623).

Una herramienta que puede favorecer el proceso de enseñanza y aprendizaje es el uso del software CmapTools para crear mapas conceptuales, el cual ha sido desarrollado por el Institute for Human and Machine Cognition y que permite a los alumnos y profesores "crear mapas conceptuales sobre un tema específico, colaborando electrónicamente en su construcción y complementándolos con imágenes, videos, textos, simulaciones, etc." (Ríos, et ál., 2007: 5).

Existen mapas conceptuales creados por expertos que son navegados por los alumnos, 
quienes pueden participar de forma conjunta en su elaboración, promoviendo el aprendizaje colaborativo y eliminando las barreras del espacio y el tiempo. Se puede lograr el aprendizaje debido a las posibilidades que ofrecen las TIC para representar, procesar, transmitir y compartir información.

"La información se convierte en conocimiento y el acceso a la información da lugar al aprendizaje cuando actuamos sobre ella, la procesamos, la organizamos, nos la apropiamos, la utilizamos y la confrontamos con otros; en suma, cuando somos capaces de darle significado y sentido" (Coll, 2003:11)

Asimismo, al utilizar el CmapTools en el proceso de enseñanza y aprendizaje es necesario rescatar los usos educativos que desde el constructivismo sociocultural se plantean: el papel del mediador; la importancia del diseño pedagógico, que junto con el uso de la tecnología permitirá la aplicación de un diseño tecnopedagógico que busque la colaboración, donde es clave la "dinámica interna de la actividad conjunta que despliegan sus miembros en torno a los contenidos y tareas de aprendizaje" (Coll, et ál., 2007b: 6).

A partir de la perspectiva constructivista de orientación sociocultural, en este aporte al proceso educativo se pretende describir la contribución que el software CmapTools -visto como un instrumento psicológico- puede aportar al proceso de enseñanza y aprendizaje, concretamente al ajuste de la ayuda del docente, así como su participación en el proceso de autorregulación individual y grupal en situaciones de aprendizaje colaborativo.

Vygostsky define los instrumentos psicológicos como:

"...recursos para dominar los procesos mentales. Se consideran artificiales y de origen social en lugar de orgánicos y de origen individual. Vygostsky dio los siguientes ejemplos de instrumentos psicológicos: la lengua, los diversos sistemas para contar, las técnicas mnemónicas, los sistemas de símbolos algebraicos, las obras de arte, la escritura, los esquemas, los diagramas, los mapas, los dibujos técnicos y todo tipo de signos convencionales" (Daniels, 2003: 33)

Los instrumentos psicológicos permiten al alumno comunicar y representar la información y contrastarla con otros, facilita que la atención se centre en las características y propiedades del entorno simbólico o semiótico. Esto debido a que las personas utilizan los sistemas de símbolos, para la regulación de los procesos mentales y la actividad con otras personas con las que se relacionan (Coll, 2003; Kozulin, 2000).

\section{MARCO TEÓRICO}

Desde la perspectiva constructivista el aprendizaje escolar se da a partir de la interacción entre el profesor que ayuda a los alumnos a construir significados y a atribuir sentido a lo que hacen y aprenden, los alumnos que aprenden y los objetivos que son objeto de enseñanza y aprendizaje, es decir, desde el triángulo interactivo (Figura 1).

"Lo que los alumnos aportan al acto de aprender, su actividad mental constructiva, es un elemento mediador entre la enseñanza del profesor y los aprendizajes que llevan a cabo" (Coll y Martín, 2001: 179). Por tanto, el proceso de enseñanza es un proceso de ajuste para la ayuda educativa que brinda el docente,

"en donde la ayuda se ajusta y adapta al proceso de construcción que llevan a cabo los alumnos o aprendices; es decir, la que, teniendo en cuenta de manera dinámica lo que los alumnos son capaces de comprender y hacer en cada momento, cambia en tipo y grado en función de las contingencias, da apoyo en aquello que se necesita y cuando se necesita, y lo retira globalmente de manera progresiva para facilitar el uso autónomo y funcional de lo aprendido en 


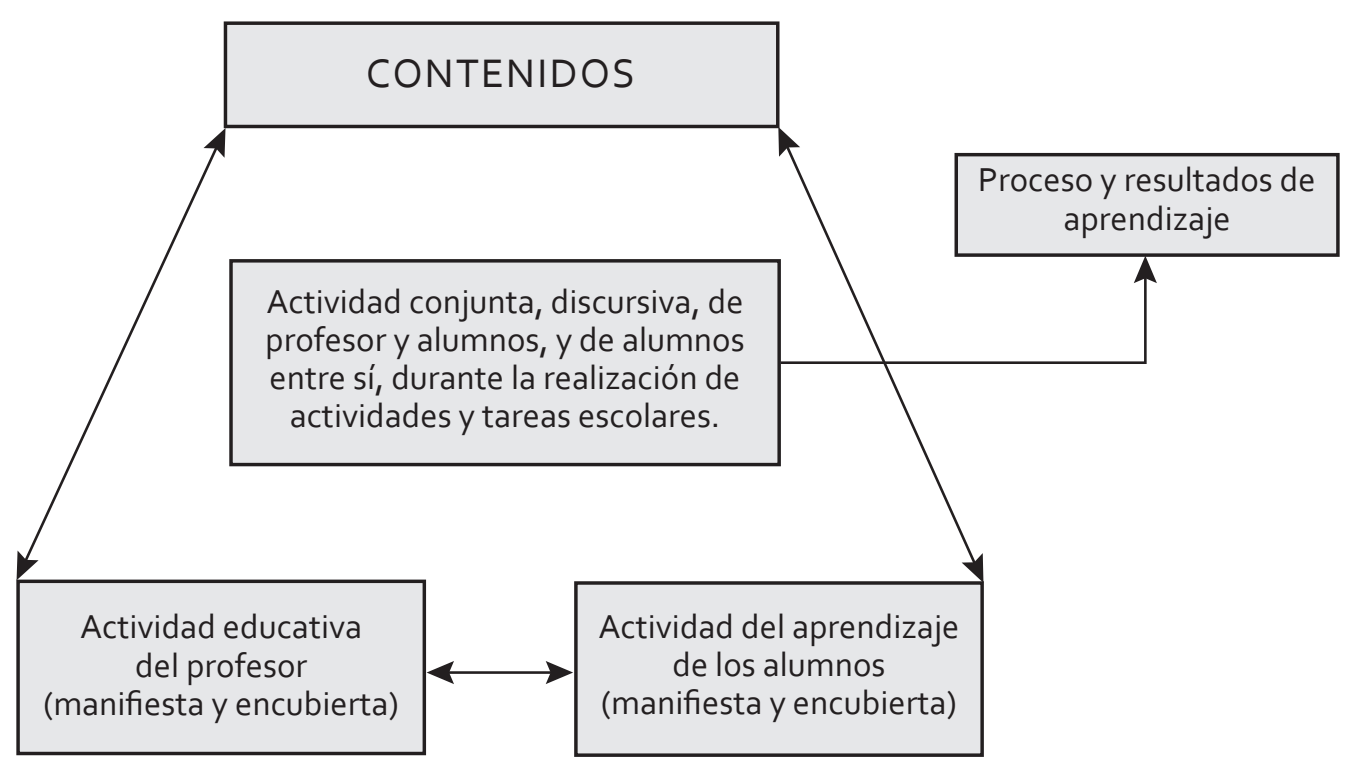

Figura 1. El aula como contexto de enseñanza y aprendizaje: un esquema constructivista.

Fuente: Coll, C. y Solé, I. Enseñar y aprender en el contexto del aula. Psicología de la educación escolar. Alianza, Madrid, 2001, p.p 357-386.

situaciones cada vez más variadas y complejas". (Coll, et ál., 2008: 36).

El ajuste de la ayuda supone la diversificación de los distintos apoyos y la flexibilidad en los diferentes momentos del proceso educativo y así lograr la atención a la diversidad y el aprendizaje de los alumnos de la construcción de la actividad mental cognitiva generadora de significado y sentido.

Ahora bien, para que los alumnos construyan y reconstruyan sus conocimientos, es importante considerar lo que plantean diferentes investigaciones realizadas sobre lo necesario de distinguir entre la adquisición de conceptos y el desarrollo de conocimientos sólidos y útiles, los cuales se presentan cuando los alumnos construyen significados basados en sus experiencias de interacción con otros, en donde exploran el mundo que les rodea, cuentan con contextos que promueven la cognición situada, la actividad conjunta, los procesos cognitivos, entre otros; que les permiten observar, actuar y practicar técnicas y metodologías sobre la resolución de problemas (Díaz Barriga, 2006; Collins, 2006; Brown, Collins \& Duguid, 1989; Tharp, et ál., 2002).

Con el propósito de que los alumnos construyan y reconstruyan conocimientos duraderos pueden utilizar los mapas conceptuales, estos les permitirán construir conocimientos, resolver problemas y desarrollar habilidades, enfatizando en el aprender y compartir con otros lo aprendido (Collins, 2006). Lo anterior tiene relación con la acción conjunta en la que se implican profesores y alumnos durante algún periodo, en la cual desarrollan actividades sobre cierto contenido que plasman en un mapa conceptual, el cual representa la construcción de significados que surgen a partir de la regulación y autorregulación que se manifiesta durante el proceso de elaboración, en el que la ayuda de los profesores y los compañeros es clave para experimentar la construcción de conocimientos (Cubero, 2005; Coll, 2004). 
También, los alumnos podrán, entre otros aspectos:

» Tomar conciencia de sí mismos y lograr que lo interpsicológico se vuelva intrapsicológico (zona de desarrollo próximo) a partir de la interacción con compañeros y docentes (Wertsch, 1988).

Utilizar los sistemas o signos culturales de los que se han apropiado (las herramientas o instrumentos psicológicos).

» Adquirir información en un contexto significativo y relacionarla con sus conocimientos previos, a fin de desarrollar un entendimiento conceptual más amplio.

»Aprender en contextos que promuevan la comprensión del significado de conceptos y los hechos en sí mismos, asociaciones y solución de problemas (Collins, 2006).

"Realizar tareas y problemas que son escogidos para ilustrar el poder de ciertas técnicas y métodos que pueden practicarse en diferentes situaciones (Brown \& otros, 1989; Collins, 2006).

Lo anterior facilitará el aprendizaje y permitirá al docente realizar el ajuste o retiro de las ayudas educativas a partir de los avances de los alumnos (andamiaje) en cada momento del proceso de enseñanza y aprendizaje, secuenciando las tareas de lo más simple a lo más complejo (Pea, 1993).

\section{- Mapa conceptual. Descripción de la herramienta y su uso educativo}

Desde hace quince años aproximadamente el Institute for Human and Machine Cognition (IHMC) ha estado desarrollando CmapTools, "un ambiente de software cliente-servidor que facilita la construcción y el compartir mapas conceptuales" programado en Java y cuyo propósito es apoyar la colaboración y el compartir (Novak \& Cañas, 2007: 1). Cualquier persona puede crear una carpeta y construir, copiar y/o publicar mapas conceptuales o almacenarlos en los servidores CmapServers, ya que muchos son públicos y permiten a cualquier usuario publicar mapas y utilizar los recursos libremente. Los mapas pueden enlazarse con recursos (fotos, imágenes, gráficos, videos, cartas, tablas, textos, páginas $u$ otros mapas) situados en cualquier lugar de Internet.

Desde la concepción constructivista sociocultural, la utilización de las tecnologías de la información en la elaboración de mapas conceptuales, pretende transformar las relaciones entre los tres elementos del triángulo interactivo (alumnos, profesor y contenidos) y, por ende, las prácticas educativas.

"No son las TIC, sino las actividades que llevan a cabo profesores y estudiantes gracias a las posibilidades de comunicación, intercambio y acceso y procesamiento de la información que les ofrecen las TIC, donde hay que buscar las claves para comprender y valorar el alcance de su impacto sobre la educación [...] incluido su eventual impacto sobre la mejora de los resultados del aprendizaje" (Coll, 2004: 5).

¿Qué son los mapas conceptuales? Desarrollados por Novak en la década de los ochenta, son una representación gráfica que muestra una serie de conceptos unidos a través de palabrasenlace para formar proposiciones, es decir, oraciones que tienen un valor de verdad (Ramírez y Sanabria, 2004: 1).

Para elaborar un mapa es necesario que el mediador y los alumnos tengan claro su propósito, reciban información por parte del docente $y$ hagan lecturas útiles sobre el tema por desarrollar, a partir de las cuales se habrá de formular una pregunta que busque su respuesta en una investigación realizada por los alumnos, para lo cual es necesario subrayar las palabras 
más importantes -lo que permitirá la construcción del mapa-. A las palabras seleccionadas se les debe dar un orden jerárquico y establecer las relaciones entre ellas, después de lo cual, y utilizando la simbología que ofrece el CmapTools, se empieza a elaborar el mapa, se ubican las palabras y se relacionan entre sí por medio de conectores y flechas. La construcción de mapas debe ser vista como "una actividad creativa, en la cual el estudiante debe hacer un esfuerzo para aclarar significados por medio de identificar los conceptos importantes, relaciones y estructura dentro de un dominio específico de conocimiento" (Novak \& Gowin, 1984, citados por Novak \& Cañas, 2007: 3).

En la Figura 2 se aprecia los símbolos que se usan en la elaboración de Mapas Conceptuales.
Cabe señalar que en la construcción de un mapa conceptual el producto final no es lo único importante, también lo es el proceso que los alumnos realizan y el acompañamiento del docente, ya sea de forma sincrónica o asincrónica, pues si se opta por esta última opción todo el proceso de elaboración queda grabado y el mediador puede realizar sus aportes.

"Las TIC son un instrumento para amplificar las posibilidades de seguimiento $y$ ayuda ajustadas al proceso de aprendizaje de los alumnos. Frente a los modelos que -implícita o explícitamente- plantean que la incorporación de las TIC a los procesos de enseñanza y aprendizaje conlleva una reducción del papel del profesor o incluso su desaparición, este planteamiento
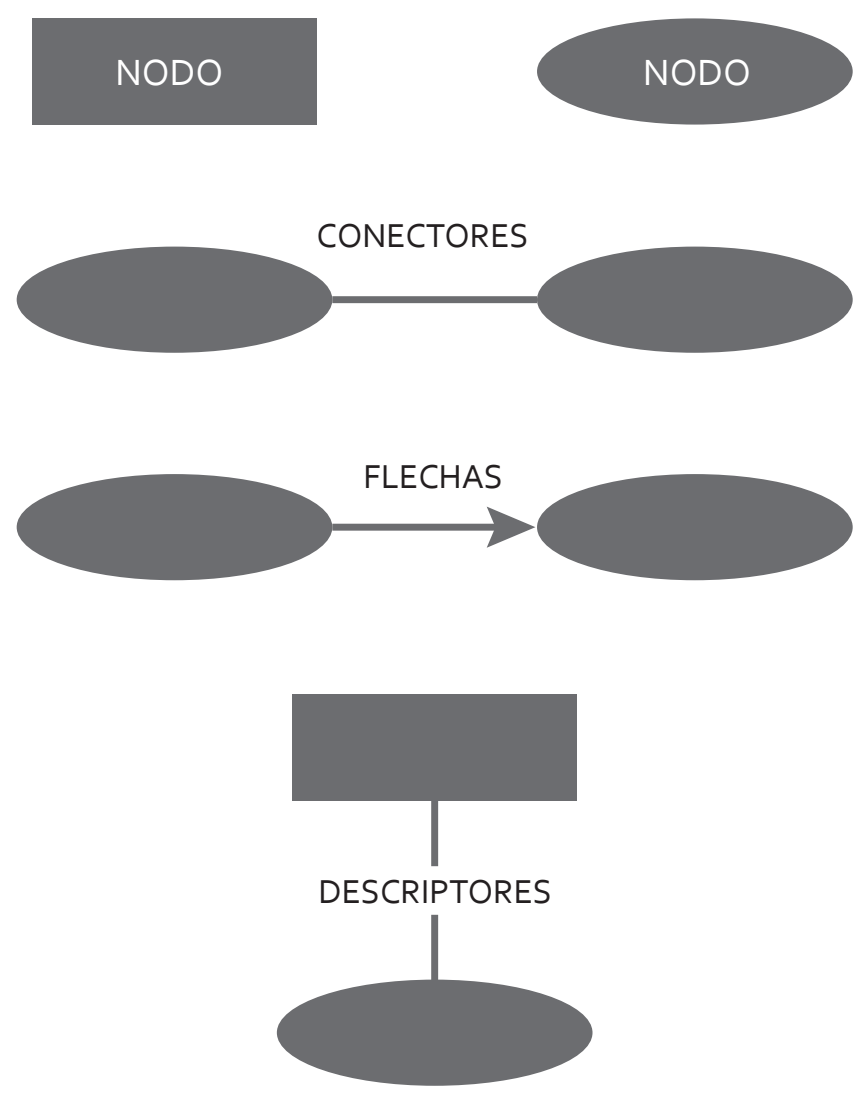

Los nodos se utilizan
para agregar palabras
clave, ideas o conceptos

Los conectores se usan para establecer conexiones o relaciones

Las flechas ayudan a establecer la direccionalidad de las relaciones

Los descriptores son la palabra o palabras que describen la conexión

Figura 2. Simbología de los mapas conceptuales. 
considera que algunas de las posibilidades más interesantes del uso de las TIC en la educación tienen que ver con su capacidad para aumentar la aptitud de seguimiento y apoyo contingente por parte del profesor al proceso de trabajo y estudio de los alumnos." (Coll, 2004; Onrubia, 2005 citados por Coll et ál., 2006: 33).

Al utilizar CmapTools para la elaboración de mapas conceptuales, el docente debe aspirar a promover el aprendizaje autorregulado, en el que intervienen "factores de muy diversa índole, cognitivos y metacognitivos, afectivos, motivacionales y volitivos" (Pintrich, 2000, citado por Coll et ál., 2007a: 785), que se interrelacionan de forma flexible y dinámica para generar, mantener y modificar el comportamiento en correspondencia con los objetivos planteados.

El aprendizaje autorregulado tiene como objetivo que las personas aprendan por sí mismas, dirijan su aprendizaje, sean responsables, tengan una visión sistémica al analizar las interrelaciones del conjunto, entiendan los problemas de forma no lineal, tengan capacidad para trabajar en equipo, reestructurar modelos mentales, aprender de la experiencia y de los errores y desarrollar la creatividad y la innovación.

El aprendizaje autorregulado consiste en procesos reguladores que se organizan en cuatro fases (Pintrick, 200ob, citado por Torrano y González, 2004: 3):

"Planificación: los alumnos deben establecer las metas que desean alcanzar o el objetivo específico a lograr con la elaboración del mapa conceptual. Para esto es importante la activación del conocimiento previo sobre la materia y del conocimiento metacognitivo, pues los alumnos deben tener claros los saberes y habilidades que necesitan para dar respuesta a la tarea por realizar.

Auto-observación: se incluyen las actividades que ayudan al alumno a tomar conciencia del estado de su cognición, su motivación, su afecto, su uso del tiempo y del esfuerzo, así como de las condiciones de la tarea y del contexto.

Control: a la luz de los resultados de la fase anterior, se ponen en marcha las actividades de control, que engloban la selección y utilización de estrategias para controlar el pensamiento, la motivación y el afecto.

Evaluación: Abarca los juicios y las evaluaciones que los alumnos realizan acerca de su ejecución en la tarea, comparándola con los criterios previamente establecidos por ellos (o por el profesor).

Las cuatro fases descritas representan una secuencia general por donde el alumno avanza mientras realiza los mapas conceptuales, pero no están jerárquica o linealmente estructuradas y pueden darse de forma simultánea y dinámica, produciendo una múltiple interacción entre los diferentes procesos y componentes incluidos en ellas".

Los nuevos descubrimientos en el aprendizaje de las ciencias han llevado a nuevas formas de entender cómo aprenden las personas y la importancia de hacerlo junto a otros, para lo cual es fundamental la construcción activa, donde los alumnos construyen y reconstruyen su mapa conceptual a partir de conocimientos previos y experiencias nuevas (Krajcik, \& Blumenfeld, 2006).

Realizar el mapa en forma conjunta permite desarrollar la dimensión social del aprendizaje de los alumnos, donde la relación entre iguales facilita la discusión sobre algún tema específico, dar soluciones y defender su decisión. El grupo de trabajo se define como "un sistema social complejo que desarrolla múltiples funciones interdependientes [...] y que las tareas que realizan son influidas por aspectos individuales y que intervienen en el trabajo grupal [...]" (Sosa, et ál., 2006: 24). 
Los alumnos no colaboran entre sí de manera natural en el aula, los profesores necesitan ayudarlos a desarrollar "habilidades de colaboración, incluyendo la conversación en turnos, escuchar y respetar las opiniones de otros" (Krajcik, \& Blumenfeld, 2006: 321). Además, deben enfrentar el cambiar la cultura del aula desde el estilo de transmisión y adquisición que los alumnos esperan, al trabajo y la respuesta grupales, donde cuenten con el apoyo del docente, pero la responsabilidad esté centrada en los alumnos.

Es oportuno que cuando los alumnos tengan que trabajar algún proyecto y deban elaborar un mapa conceptual, los docentes fomenten, en el proceso de enseñanza y aprendizaje (proceso de autorregulación), la utilización de CmapTools. Además, deben tomarse en cuenta los siguientes puntos por realizar:

» Plantearse una pregunta, un problema por resolver.

»Explorar la pregunta participando en búsquedas auténticas y situadas.

» Involucrarse, junto a los profesores, en actividades de colaboración para hallar soluciones a la pregunta.

» Utilizar CmapTools como un medio que facilite el aprendizaje individual y grupal, a partir del trabajo asincrónico y sincrónico.

»Crear una serie de productos tangibles que traten la pregunta. Éstos son artefactos compartidos, representaciones externas del aprendizaje de la clase que pueden ser accesibles al público (Blumenfeld, 1991; Krajcik, 1994; Krajcik, Czerniak y Berger, 2002, citados por Krajcik, \& Blumenfeld, 2006).

Al implementar las acciones antes mencionadas, los alumnos estarán trabajando un proceso de aprendizaje de autorregulación individual y grupal en situaciones de colaboración, en el cual intervienen factores cognitivos, metacognitivos, afectivos y motivaciones que les permitirán asumir roles y tener un aprendizaje situado.

\section{CONCLUSIONES}

Si bien la metodología de mapas conceptuales ha sido desarrollada desde los años ochenta, no es sino hasta hace 15 años aproximadamente que se ha creado CmapTools, que permite promover el trabajo colaborativo siempre y cuando se utilice dentro de un diseño tecnopedagógico.

La concepción constructivista sociocultural del aprendizaje plantea que las TIC deben ser utilizadas como herramientas semióticas o instrumentos psicológicos que pueden influir en los procesos de construcción de significados y de atribución de sentido que llevan a cabo las personas durante los procesos de enseñanza y aprendizaje.

La utilización de las TIC en ningún momento desplaza al docente, todo lo contrario, le permite dar seguimiento a sus alumnos y ajustar la ayuda durante el proceso de trabajo. El ajuste de la ayuda supone la diversificación de los distintos apoyos y la flexibilidad en los diferentes momentos del proceso educativo, de esta forma se logra la atención a la diversidad y el aprendizaje de los alumnos de la construcción de la actividad mental cognitiva generadora de significado y sentido.

Con el objetivo de que los alumnos construyan y reconstruyan conocimientos duraderos, la utilización de los mapas conceptuales les permitirá construir conocimientos, resolver problemas y desarrollar habilidades, con base en el aprender y compartir con otros lo aprendido.

\section{REFERENCIAS}

Brown, J. S.; Collins, A. \& Duguid, P. (1989). Situated cognition and the culture of learning. Educational Researcher, 18(1), 32-42. 
Coll, C. (2001). Constructivismo y educación: la concepción constructivista de la enseñanza y el aprendizaje. En: Coll, C.; Palacios, J.; Marchesi, A. Desarrollo psicológico y educación. Vol. 2. Psicología de la educación escolar Madrid: Alianza.

Coll, C. (2003). La potencialidad de las tecnologías de la información y de la comunicación para el aprendizaje. En Coll, C; Reeves, T; Hirumi, A y Peters O. Del docente presencial al docente virtual. Procesos formativos de enseñanzaaprendizaje on-line. Universidad Oberta de Catalunya. Consultado el 20 de setiembre de 2010, http://etic-grupog.wikispaces.com/file/ view/PROCESO+FORMATIVOS+DE+ENSE\%C 3\% 1 IANZA+APRENDIZAJE+ONLINE.pdf.

Coll, C. (2005a).Una aproximación socio-constructivista a la enseñanza y el aprendizaje en entornos virtuales: implicaciones para el diseño y la investigación. Ponencia presentada en el Programa de doctorado sobre Intervención en Pedagogía y Psicopedagogía. Facultad de Ciencias de la Educación. Universidad de Granada. Consultado el 7 de abril de 2009, http://www. psyed.edu.es/prodGrintie/articulos/ TM_RC_ IdG_DisenoPropDocent_07.pdf

Coll, C. (2005b) Ayudar a aprender con las TIC: sobre los usos de la tecnología en la educación formal. Conferencia presentada Congrés Multimedia Educativa: Els reptes educatius de la societat digital. 29 de juny -1 de julio de 2005, Universitat de Barcelona. Consultado el 22 de setiembre 2010, http://www.psyed.edu.es/ prodGrintie/conf/CC_VMME_05.pdf

Coll, C. (2004). Psicología de la educación y prácticas educativas mediadas por las tecnologías de la información y la comunicación. Una mirada constructivista. Revista Sináctica, pp. 1-24. Consultado el 28 de setiembre de 2010, http:// giddetunam.org/prod/articulos/practicas_mediadas.pdf

Coll, C. y Martí, E. (2001). La educación escolar ante las nuevas tecnologías de la información y de la comunicación. En: Coll, C.; Palacios, J.; Marchesi, A. Desarrollo psicológico y educación. Vol. 2. Psicología de la educación escolar Madrid: Alianza.
Coll, C., Mauri, T. y Onrubia, J. (2006). Análisis y resolución de casos-problema mediante el aprendizaje colaborativo. RUSC - Revista de Universidad y Sociedad del Conocimiento. 3(2). Consultado el 5 de mayo de 2010, http://www.uoc.edu/rusc/3/2/dt/esp/coll_mauri_onrubia.pdf

Coll, C.; Rochera, M.; Mayordomo, R.; y Naranjo, M. (2007a). Evaluación continúa y ayuda al aprendizaje. Análisis de una experiencia de innovación en educación superior con apoyo de las TIC. Revista Electrónica de Investigación Psicoeducativa, No 13 Vol. 5 (3) pp. 783-804.

Coll, C., Onrubia, J. y Mauri, T. (2007b).Tecnología y prácticas pedagógicas: las TIC como instrumentos de mediación de la actividad conjunta de profesores y alumnos. Anuario de Psicología, 38(3), 377-400.

Coll, C., Onrubia, J. y Mauri, T. (2008). Ayudar a aprender en contextos educativos: el ejercicio de la influencia educativa y el análisis de la enseñanza. Revista Educación, 346, pp. 3370. Consultado el 12 de setiembre de 2010, http://www.revistaeducacion.mec.es/re346/ re346_02.pdf

Coll, C y Solé, I. (2001). Enseñar y aprender en el contexto de aula. En: Coll, C.; Palacios, J.; Marchesi, A. Desarrollo psicológico y educación. Vol. 2. Psicología de la educación escolar Madrid: Alianza.

Collins, A. (2006). Cognitive apprenticeship. En R. Keith Sawyer (Ed.), The Cambridge handbook of the learning sciences (pp. 47-60). Cambridge: Cambridge University Press.

Cubero, R. (2005). Perspectivas constructivistas: La intersección entre el significado, la interacción y el discurso. España: Editorial GRAÓ.

Daniels, H. (2003). Vygotsky y la pedagogía. Barcelona: Paidós.

Díaz Barriga, F. (2006). Enseñanza situada: Vínculo entre la escuela y la vida. México: McGraw Hill.

Kozulin, A. (2000). Instrumentos psicológicos. La educación desde una perspectiva sociocultural. Barcelona: Paidós 
Krajcik, J. S. \& Blumenfeld, Ph. C. (2006). Projectbased learning. En R. Keith Sawyer (Ed.), The Cambridge handbook of the learning sciences (pp. 317-333). Cambridge: Cambridge University Press.

López, M. (2003). Los mapas conceptuales y su uso en los curso en línea. Universidad Iberoamericana. México. Consultado el 3 de mayo de 2009 http://cmap.javeriana. edu.co/servlet/SBRead ResourceServlet? rid $=1264791256328 \_862611$ 049_2784 Revisado en mayo 2008.

Novak, J. y Cañas, A. (2007). Construyendo sobre nuevas ideas constructivistas y la herramienta Cmap Tools para crear un nuevo modelo educativo. Consultado el 14 de febrero de 2010, http://www.ihmc.us/users/acanas/Publication s/NewModelEducation/NuevoModeloEducacion.pdf.

Ramírez, M. y Sanabria, I. (2004). El mapa conceptual como elemento fundamental en el proceso de enseñanza-aprendizaje de la física a nivel universitario. Venezuela. Universidad Nacional del Táchira. Consultado el 17 de abril de 2008, http://cmc.ihmc.us/papers/cmc2004-086.pdf.

Ríos, L.; López, E.; Lescano, M. y Hernández, A.; García, A. (2007). Los mapas conceptuales, las TIC y el e-learning. En Revista Iberoamericana de Educación. Vol. 43, $\mathrm{N}^{\circ} 1$.
Tharp, R. G.; Estrada, P.; Stoll Dalton, S. y Yamauchi, L. A. (2002). Transformar la enseñanza. Excelencia, equidad, inclusión y armonía en las aulas y las escuelas. Barcelona: Paidós.

Sosa, M., Zarco, R. \& Postiglioni, A. (2006). Modelando aspectos de grupo en entornos colaborativos para proyectos de investigación. En Revista de Informática Educativa y Medios Audiovisuales. Vol. 3 (7), págs. 22-31.

Pea, R. D. (1993). Prácticas de inteligencia distribuida y diseños para la educación. En G. Salomon (Ed.), Cogniciones distribuidas. Consideraciones psicológicas y educativas (pp. 75-125). Buenos Aires: Amorrortu.

Torrano, M. y González, M. (2004) El aprendizaje autorregulado presente y futuro de la investigación. Departamento de Educación, Universidad de Navarra. Revista Electrónica de Investigación Psicoeducativa. (1). 1-34. Consultado el 9 de junio de 2010, http://wtigacion-sicopedagogica.org/revista/articulos/3/espannol/ Art_3_27.pdfww.inves

Vygotsky, L.S. (1979). El desarrollo de los procesos psicológicos superiores. Barcelona: Crítica.

Wertsch, J.V. (1988). Vygotsky y la formación social de la mente. Barcelona: Paidós. 